\title{
Nitric Oxide as a Potential Bridge Between the Metabolic and Vascular Hypotheses of Diabetic Neuropathy
}

\author{
M.J. Stevens \\ Department of Internal Medicine, and the Michigan Diabetes \\ Research and Training Center, University of Michigan, Ann Arbor, \\ USA
}

\begin{abstract}
Both metabolic and vascular factors have been invoked in the pathogenesis of diabetic neuropathy but their interrelationships are poorly understood. Both aldose reductase inhibitors and vasodilators improve nerve conduction velocity, nerve blood flow, and $\left(\mathrm{Na}^{+}, \mathrm{K}^{+}\right)$-ATPase activity in the streptozotocin diabetic rat, implying a metabolic-vascular interaction. Nitric oxide may be the 'bridge' linking these divergent hypotheses of diabetic neuropathy. We propose a model for the pathogenesis of neuropathy invoking metabolic defects both at a vascular and neurochemical level. Early after the induction of experimental diabetes, metabolic defects may lead to a decrease in synthesis of nitric oxide in either the vascular endothelium or the sympathetic ganglia leading to decreased nerve blood flow. In addition, nitric oxide may be involved in more distal defects of somatic nerve metabolism which impair the activity of the nerve $\mathrm{Na} / \mathrm{K}$-ATPase by a mechanism involving phosphoinositide signaling and diacyl glycerol and may therefore affect nerve conduction velocity independently of ischaemia. Improved understanding of the effects of hyperglycaemia on nitric oxide metabolism, may provide important clues elucidating the mechanisms underlying the pathogenesis of diabetic neuropathy.
\end{abstract}

KEY wORDS Diabetic neuropathy Nitric oxide Aldose-reductase inhibition

The aetiology of diabetic neuropathy remains unknown but evidence implicates a multifactorial pathogenesis. The potential mechanisms leading to its development are difficult to elucidate in many diabetic patients due to the pathological changes typically being well advanced and thus obscuring the early manifestations which offer aetiological clues. Most data therefore have been acquired from the diabetic rat model which develops similar functional and structural nerve defects to man. Two approaches have been utilized to explore the pathogenesis of neuropathy. Firstly, the diabetic rat has been used to characterize the metabolic and physiological defects leading to the development of experimental neuropathy and, secondly, use has been made of selective therapeutic interventions to reverse the hyperglycemia-induced abnormalities.

Numerous interventions have been shown to prevent or ameliorate nerve conduction slowing and nerve metabolic and blood flow abnormalities in the diabetic rat model. Given the diverse nature of these therapeutic interventions, can a unified hypothesis be created?

Considerable evidence implicates nerve ischaemia in the pathogenesis of diabetic nerve conduction slowing as haemodynamic and oximetric measurements in anaesthetized rats have demonstrated reduced endoneurial

Correspondence to: Dr M. Stevens, Division of Endocrinology and Metabolism. Department of Internal Medicine, University of Michigan Medical Center, 5570, MSRB II, 1150 West Medical Center Drive Ann Arbor, MI 48109-0678, USA. blood flow and oxygen tension, and increased endoneurial vascular resistance early after the induction of streptozotocin diabetes. ${ }^{1}$ Studies from the same laboratory have also demonstrated that in streptozotocin diabetic rats endoneurial blood flow and nerve conduction can be partially or completely corrected by a variety of vasodilatory agents including the ACE inhibitor lisinopril. ${ }^{2}$ Moreover, many metabolic interventions which have been demonstrated to prevent the development of nerve conduction slowing in diabetic rats may also be working through a vascular mechanism. For example, aldose reductase inhibitors (ARIs) have been shown to correct the early reduction in nerve blood flow in experimental diabetes $^{3}$ and also restore to normal the diabetes or hyperglycaemia-induced deficits in endothelium-dependent vascular relaxation. ${ }^{4}$

Although the success of vasodilatory therapeutic interventions on the reversal or prevention of nerve conduction slowing has confirmed the importance of blood flow deficits in the pathogenesis of experimental neuropathy, a number of questions remain. For example, how does diabetes precipitate a decrease in endoneurial blood flow? How does this decreased blood flow cause nerve conduction slowing? Can therapeutic interventions which fail to correct blood flow ameliorate or prevent experimental neuropathy?

We propose a model for the pathogenesis of neuropathy invoking metabolic defects both at a vascular and neurochemical level (Figure 1). These defects may mediate both the early reduction in nerve blood flow 


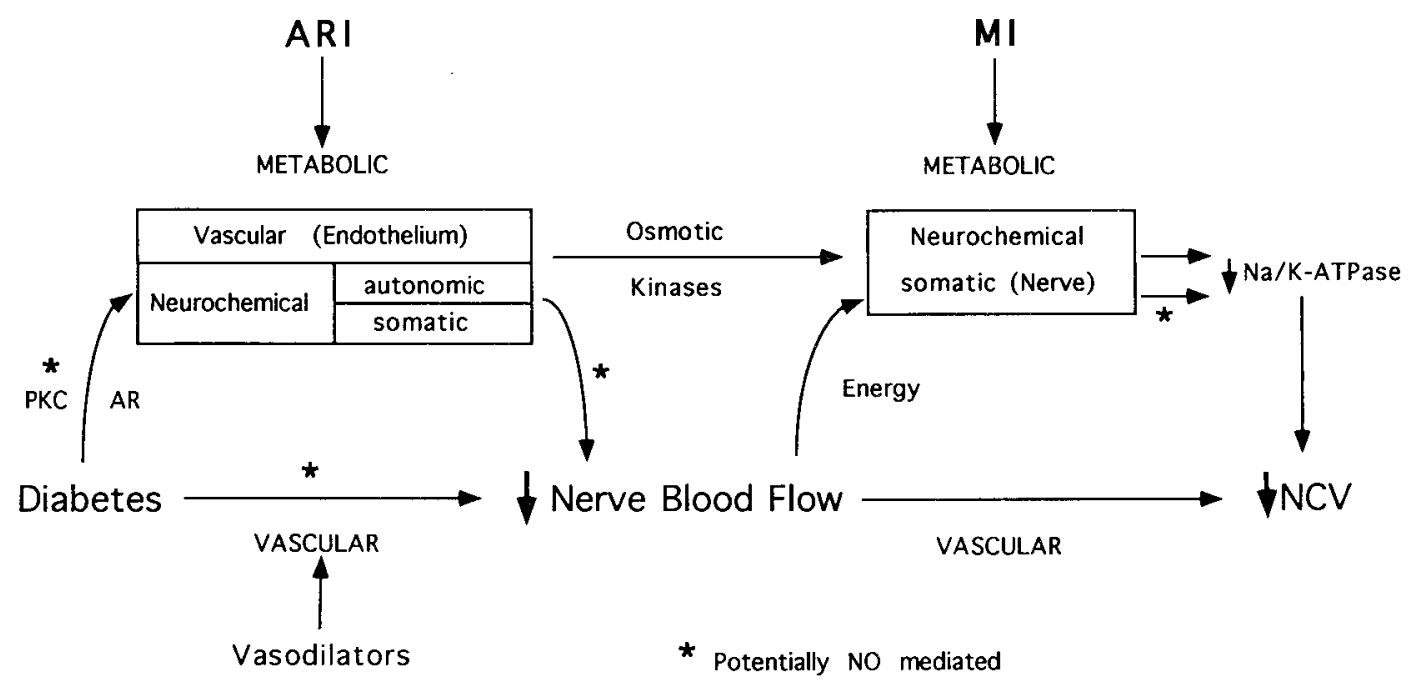

Figure 1. Model demonstrating the proposed mechanisms and sites at which metabolic and vascular abnormalities in diabetes could lead to nerve conduction slowing. Also shown are the steps which could potentially involve nitric oxide. ARI, aldose reductase inhibition; MI, myo-inositol; PKC, protein kinase $C$; $A R$, aldose reductase; NO, nitric oxide; NCV, nerve conduction velocity

and the later development of nerve conduction slowing. For example, soon after the induction of diabetes in the rat, metabolic defects in either the vascular endothelium or within the sympathetic ganglia could precipitate an increase in basal vascular tone leading to the development of nerve ischaemia. In addition, more distal defects of somatic nerve metabolism could directly effect nerve conduction velocity independently of nerve ischaemia potentially by impairing the activity of the nerve $\mathrm{Na} / \mathrm{K}$ ATPase by a mechanism involving diacyl glycerol (DAG).

Traditionally, the principal site of metabolic defects involving phosphoinositide metabolism has been the peripheral nerve trunks. It is also possible that these defects could occur in other sites such as the autonomic nervous system and lead to secondary disturbances of vascular function. ${ }^{5}$ This situation is however complex as glucose may have bidirectional effects on diacylglycerol (DAG) and protein kinase C (PKC) activity. Diabetes and/or hyperglycaemia have been reported to decrease DAG and PKC activity in some tissues including peripheral nerve ${ }^{6,7}$ while in others such as the vascular endothelium hyperglycaemia appears to increase total DAG and PKC activity. ${ }^{8}$ Our studies in human retinal pigment epithelial (RPE) cells suggest that the determining factor for the direction of DAG changes is aldose reductase activity and also the extent to which myoinositol depletion occurs. ${ }^{9}$ RPE cells with high basal aldose reductase expression demonstrate glucose-induced decrease in myo-inositol and PKC, contrasting with PKC activation in cells with low basal aldose reductase expression.

How could changes in tissue polyol pathway flux and PKC alter nerve blood flow? We propose that the bridge between these metabolic perturbations and nerve blood flow is nitric oxide. Nitric oxide (NO) is a highly reactive, short-lived radical with a broad spectrum of metabolic functions. It has been identified as a prime candidate for the endothelium-derived relaxing factor mediating vasodilatation, it mediates macrophage cytotoxicity, and plays an important role as a neuronal messenger within the CNS and may function as an inhibitory neurotransmitter. ${ }^{10,11}$

In order to see whether the beneficial effects of aldose reductase inhibitors on nerve conduction velocity involve a nitric oxide-mediated mechanism, we evaluated whether inhibition of nitric oxide synthase (NOS) with $N$-nitro-1-arginine methyl ester (L-NAME) could block the effects of an ARI on nerve conduction. Our studies in streptozotocin diabetes rats ${ }^{12}$ demonstrated that L-NAME could reverse the protective effects of the ARI sorbinil on nerve conduction slowing in diabetic rats, a finding which supported the role of $\mathrm{NO}$ in the maintenance of normal nerve function. Increased glucose flux through the polyol pathway may therefore be a proximal metabolic defect in our model of neuropathy, in that its detrimental effects on nerve conduction velocity may be principally mediated by a reduction in nerve blood flow.

However, is there evidence for a distal metabolic component that could explain nerve conduction slowing independently of nerve ischaemia? Can distal therapeutic intervention correct nerve dysfunction independently of nerve blood flow or NO? Our data in diabetic rats suggest that this is the case. ${ }^{12}$ For example, the beneficial effects of myo-inositol on nerve conduction slowing are thought to be mediated by correction of the nerve $\mathrm{Na}$ / K-ATPase. ${ }^{13,14}$ Our studies have shown that the protective effects of myo-inositol (MI) supplementation on nerve conduction velocity are not blocked by NOS inhibitors. ${ }^{12}$ Therefore as L-NAME appears to block the beneficial effects of an ARI, but not MI supplementation, and MI 
is not thought to mediate nerve blood flow, metabolic intervention aimed at correcting distal defects in nerve metabolism can correct nerve conduction slowing independently of blood flow.

Although the effects of $\mathrm{NO}$ in the preservation of nerve function are thought to be primarily mediated by a vascular mechanism, some data support a more distal metabolic role. For example, the reproduction of the glucose-induced decrease in rabbit aortic $\mathrm{Na} / \mathrm{K}$-ATPase by an NOS inhibitor ${ }^{15}$ supports the concept that $\mathrm{NO}$ can directly regulate $\mathrm{Na} / \mathrm{K}$-ATPase activity. Moreover, our studies have demonstrated that diabetic nerve $\mathrm{Na}$ / K-ATPase activity can be reproduced in nondiabetic rats given L-NAME for 3 months. ${ }^{12}$ Therefore $\mathrm{NO}$ could be modulating nerve function at both a proximal vascular level, and a more distal metabolic site.

However, how does the polyol pathway and hyperglycaemia alter NO metabolism? In mammalian CNS and endothelial cells, $\mathrm{NO}$ is generated by the enzymatic conversion of L-arginine to citrulline by $\mathrm{NO}$-synthase which in these tissues is a calcium-calmodulin-dependent constitutive isoform (cNOS). ${ }^{10,16,17}$ This cNOS generates low levels of NO which in vascular tissue modulates basal vascular tone by interacting with and activating heme-containing soluble guanylate cyclase in smooth muscle generating CGMP. In macrophages and smooth muscle, however, considerably higher levels of $\mathrm{NO}$ are also produced via a calcium-calmodulin-independent cytokine-inducible isoform (iNOS) which may promote cytokine-mediated cytotoxicity.

Glucose-induced alterations in PKC activity are proposed to modulate the activity of cNOS by direct phosphorylation. ${ }^{16,17}$ Controversy however surrounds the relationship between PKC and nitric oxide synthase. For example, PKC activation has been reported to both stimulate cNOS activity ${ }^{18}$ and also to decrease cNOS activity. ${ }^{17,19}$ We propose that flux through the polyol pathway could deleteriously affect $\mathrm{NO}$ synthesis by two mechanisms. Firstly, we have proposed that as aldose reductase and NOS share NADPH as an obligate cofactor, enhanced flux through the polyol pathway could consume $\mathrm{NADPH}$ thereby limiting its availability for the synthesis of NO. ${ }^{5,12}$ A second possibility is that decreased PKC activity in diabetes may reduce nitric oxide synthesis. Therefore accumulating evidence links NO depletion to nerve conduction slowing in diabetes. However, at what tissue site could these derangements be occurring?

Although local vascular NO depletion may directly contribute to nerve ischaemia, the potential of NO as an inhibitory neurotransmitter and its role in the maintenance of blood pressure via modulating sympathetic tone suggest another potential mechanism. The acute reduction in nerve blood flow in diabetes may be mediated by increased sympathetic tone, as adrenergic sympathectomy restores both nerve blood flow and nerve conduction to normal levels in acute experimental diabetes. ${ }^{1}$ If $\mathrm{NO}$ is functioning as an inhibitory neurotransmitter, it is tempting to speculate that post-synaptic neuronal NO might dampen presynaptic acetylcholine release thereby modulating peripheral sympathetic tone.

In summary, therefore, NO may be the 'bridge' linking the metabolic and vascular hypotheses of diabetic neuropathy. Early after the induction of diabetes, metabolic defects may lead to a decrease in synthesis of NO in either the vascular endothelium or the sympathetic ganglia leading to decreased nerve blood flow. In addition, NO may be involved in more distal defects of somatic nerve metabolism which impair the activity of the nerve $\mathrm{Na} / \mathrm{K}$-ATPase and may therefore affect nerve conduction velocity independently of ischaemia. Improved understanding of the effects of hyperglycemia on NO metabolism, may provide important clues elucidating the mechanisms underlying the pathogenesis of diabetic neuropathy.

\section{References}

1. Cameron NE, Cotter MA, Low PA. Nerve blood flow in early experimental diabetes in rats: relation to conduction defects. Am / Physiol 1991; 261: E1-E8.

2. Cameron NE, Cotter MA, Robertson S. Angiotensin converting enzyme inhibition prevents development of muscle and nerve dysfunction and stimulated angiogenesis in streptozotocin-diabetic rats. Diabetologia 1992; 35: 12-18.

3. Yasuda $\mathrm{H}$, Sonobe $M$, Yamashita $M$, Terada $M$, Hatanaka 1, Huitian $Z$, et al. Effect of prostaglandin $E_{1}$ analogue TFC 612 on diabetic neuropathy in streptozocin-induced diabetic rats: comparison with aldose reductase inhibitor ONO 2235. Diabetes $1989 ;$ 38: 832-838.

4. Cameron NE, Cotter MA. Impaired contraction and relaxation in aorta from streptozotocin-diabetic rats: role of polyol pathway. Diabetologia 1992; 35: 1011-1019.

5. Greene DA, Sima AAF, Stevens MJ, Feldman EL, Killen PD, Henry DN, et al. Aldose reductase inhibitors: An approach to the treatment of diabetic nerve damage. Diabetes Metabolism Reviews 1993; 9: 189-217.

6. Zhu $X$ and Eichberg J. 1,2-Diacylglycerol content and its arachidonyl-containing molecular species are reduced in sciatic nerve from streptozotocin-induced diabetic rats. I Neurochem 1990B; 55: 1087-1090.

7. Kim ), Rushovich EH, Thomas TP, Ueda T, Agranoff BW, Greene DA. Diminished specific activity of cytosolic protein kinase $C$ in sciatic nerve of streptozotocin-diabetic rats, and its correction by dietary myo-inositol. Diabetes 1991; 40: 1545-1554.

8. Inoguchi T, Battan R, Handler E, Sportsman IR, Heath $W$, King GL. Preferential elevation of protein kinase $C$ isoform bll and diacylglycerol levels in the aorta and heart of diabetic rats. Proc Natl Acad Sci USA 1992; 89: 11059-11063.

9. Thomas TP, Feldman EL, Nakamura J, Kato K, Lien M, Stevens MI, et al. Ambient glucose and aldose reductaseinduced myo-inositol depletion modulate basal and carbachol-stimulated phosphoinositide metabolism and diacylglycerol accumulation in human retinal pigment epithelial cells in culture. Proc Natl Acad Sci USA 1993; 90: 9712-9716.

10. Moncada S, Palmer RMJ, Higgs EA. Nitric oxide: physiology, pathophysiology and pharmacology. Pharmacol Rev 1991; 43: 109-141.

11. Garthwaite J. Glutamate, nitric oxide and cell-cell 
signalling in the nervous system. Trends Neurosci 1991; 14: $60-67$.

12. Stevens MI, Dananberg I, Feldman EL, Lattimer SA, Kamijo $M$, Thomas TP, et al. The linked roles of nitric oxide, aldose reductase and $\left(\mathrm{NA}^{+}, \mathrm{K}^{+}\right)$-ATPase in the slowing of nerve conduction in the streptozotocin diabetic rat. I Clin Invest 1994; 94: 853-859.

13. Greene DA, Lattimer SA. Impaired rat sciatic nerve sodium-potassium adenosine trisphosphatase in acute streptozotocin diabetes and its correction by dietary myo-inositol supplementation. / Clin Invest 1983; 72: 1058-1063.

14. Greene DA, Lattimer SA, Sima AAF. Sorbitol, phosphoinositides, and sodium-potassium-ATPase in the pathogenesis of diabetic complications. N Eng/ / Med 1987; 316: 599-606

15. Gupta S, Sussman I, McArthur CS, Tornheim K, Cohen
RA, Ruderman NB. Endothelium-dependent inhibition of $\mathrm{Na} / \mathrm{K}$-ATPase activity in rabbit aorta by hyperglycemia. I Clin Invest 1992; 90: 727-732.

16. Bredt DS, Snyder SH. Isolation of nitric oxide synthase, a calmodulin-requiring enzyme. Proc Natl Acad Sci 1990; 87: 682-685.

17. Bredt DS, Ferris CD. Synder SH. Nitric oxide synthetase regulatory sites. I Biol Chem 1992; 267: 10976-10981.

18. Nakane M, Mitchel L, Forstermann U, Murad F. Phosphorylation by calcium calmodulin-dependent protein kinase II and protein kinase $\mathrm{C}$ modulates the activity of nitric oxide synthetase. Biochem Biophys Res Commun 1991; 180: 1396-1402.

19. Murthy KS, Jin J.-G, Makhlouf GM. Inhibition of nitric oxide synthase activity in dispersed gastric muscle cells by protein kinase C. Am J Physiol 1994; 266: G161-G165. 